\title{
Application of Type-1 and Type-2 Fuzzy Logic Controller for the Real Swarm Robot
}

\author{
https://doi.org/10.3991/ijoe.v15i06.10075 \\ Ade Silvia Handayani, Nyayu Latifah Husni, Siti Nurmaini ( $\left.{ }^{\varpi}\right)$, Irsyadi Yani \\ Politeknik Negeri Sriwijaya, Palembang, Indonesia \\ siti_nurmaini@unsri.ac.id
}

\begin{abstract}
Navigation is one of the typical problem domains occurred in studying swarm robot. This task needs a special ability in avoiding obstacles. This research presents the navigation techniques using type 1 fuzzy logic and interval type 2 fuzzy logic. A comparison of those two fuzzy logic performances in controlling swarm robot as tools for complex problem modeling, especially for path navigation is presented in this paper. Each hierarchical of fuzzy logic shows its advantages and disadvantages. For testing the robustness of type-1 fuzzy logic and interval type-2 fuzzy logic algorithms, 3 robots for the real swarm robot experiment are used. Each is equipped with one compass sensor, three distance sensors, and one X-Bee communication module. The experimental results show that type-2 fuzzy logic has better performance than type-1 fuzzy logic.
\end{abstract}

Keywords-Interval type 2 fuzzy logic, navigation, type 1 fuzzy logic, swarm robot

\section{$1 \quad$ Introduction}

One of important research in system swarm robot is how to coordinate multi robot in that systems. An intelligence of each individual robot in the swarm in interacting among them really depends on their environment. Each of them should be able to adapt their behavior to the situation and condition that they met. Therefore, the main function of their intelligence is used for adapting their position to the environment. Some of the tasks concerning to the environment that they should handle are: navigating [1][2], foraging [3], formation controlling and formation forming [4], aggregating [5], sorting [6], and collective construction [7].

In the navigation task, the individual robots of swarm should be able to help one another to reach the target, i.e. by finding the optimal or suboptimal path. This task of course needs a special ability, especially in avoiding obstacles [8]. The environment that the robots employ in conducting the navigation task can be classified into 3 types, namely:

1. Known environment (conditioned) [9]

2. An unknown environment [8] [10] 
3. A dynamically changing environment [11][12]. It is contradictive with the known environment, the navigation through unknown and dynamic environments needs an approach that can control the occurrence of an uncertain situation [10][13].

One approach that can control the uncertain situation in swarm robot application is the use of fuzzy logic. This technique can shorten the time, avoid the obstacles and smooth the movement of the robot in a very complex system [14]. This method is one of the most famous intelligent computation [15].

Basically, besides fuzzy logic, there are a lot of approaches that are used as intelligent computation methods, for instances: ant colony optimization algorithm [16], artificial neural network, neuro-fuzzy [17], particle swarm optimization [14], genetic algorithm [18][19], simulated annealing algorithm [20][21].

In conducting the navigation system in swarm robot, the main framework from the fuzzy reasoning allows fuzzy sets of type 1 membership functions to handle uncertainty [22][23]. However, in many cases, there was no information about the sufficient membership function shapes. Therefore, using type 2 fuzzy sets becomes important. Type 2 fuzzy logic is a more general formulation that used fuzzy membership functions with additional dimension [24][25]. It supplies an additional degree of freedom from lacking information and to handle uncertainties.

In previous research, Type 1 Fuzzy Logic System (T1FLS) has been developed in handling the navigation of some robots [26], whereas the application of Interval Type 2 Fuzzy Logic System (IT2FLS) was still rare. IT2FLS can handle uncertainties system better than T1FLS. Therefore, the IT2FLS began to take over T1FLS. In the paper of Hagras [27], it presented autonomous mobile robots with reactive control architecture which is based on IT2FLS to carry out basic navigation behaviors and coordination.

The main difference between T1FLS and IT2FLS is in the last process, at least one membership functions from the rule base of type 1 also the membership function from the type 2 [25]. Therefore, the output of the inference engine outputs is type-2 [28].

In the case of IT2FLS, the cost of computing reduction types is the main point [22][23]. The controller really relies on the reduction type and the defuzzification process. So, reduce the computational burden and the resources to be implemented these two stages are very comfortable. In addition, there are other applications of IT2FLS where the hardware and software platforms intricacy must be reduced to guarantee the fastest possibility of fuzzy inferences [29][30].

The study of a fuzzy logic system for mobile robot navigation has been done in several types of research over the past few years. However, it is rarely discussed in the swarm robot navigation. In this paper, the main focus is a comparative study of navigation techniques development in swarm robots, using type 1 and intervals type 2 fuzzy logic controller. This method has been showed and proven to perform better than established controllers. The output of a review of previous research has proven, that they are distinguished in numerical terms in finding good results from a specified search space, no matter how many parameters they trace. 


\section{Navigation Techniques in Swarm Robots}

\subsection{Using type 1 fuzzy logic controller}

In navigating each individual swarm robot, it is necessary to control their orientation and speed by using sensor data. Control of robots using established control algorithms has not been satisfactory, as far as the robot is in a complex environment [31]. Fuzzy logic is either the efficient method for the navigation in mobile robot in an uncertain environment.

The use of the fuzzy logic method in the navigation task has been analyzed by a lot of previous studies. In overcoming the obstacle avoidance and stabilization of the position of mobile robot wheel problem Faisal et al [32] has designed sensor-based fuzzy sensor wireless for mobile robot navigation tasks between static and moving barriers. While in the paper [33], it can be seen that there were design and implementation of the fuzzy hybrid architecture for intelligent navigation systems and mobile robot control in avoiding obstacles in static and dynamic environments. Just as in the case of robot football, fuzzy logic is very important, applied to individual robot behaviors and actions, especially for obstacle avoidance and achieving targets [34][35]. In research [8], Algabri et al. have designed two fuzzy logic behaviors for mobile robot navigation i.e., behavior to achieve targets and avoid obstacles with different scenarios. However, it is important to pay attention to the development of this architecture, that is for the same path-planning problem.

Currently, the fuzzy logic system mostly included four components: fuzzifier, rulebase, inference engine, and defuzzifier (shown Fig.1). Accurate input variables became input grades called as fuzzy variables converted by the fuzzification and determined the degree of which crisp values.

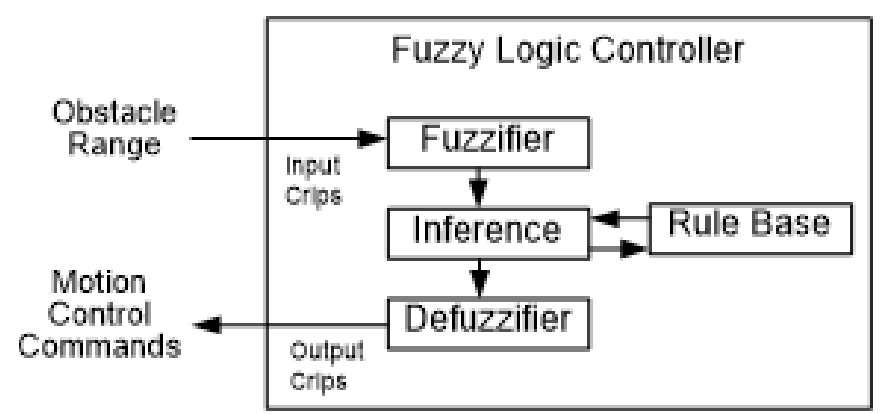

Fig. 1. Structure Type 1 Fuzzy Logic Control on Swam Robot

Included into which fuzzy that is generated by collecting information from the experts or from the trial and error. The mechanism for modeling to show the input amount determined by an expert is called inference. Fuzzy variables converted by the 
defuzzification to an accurate output variable and it converted back a fuzzy membership value into a crisp value.

\subsection{Using type 2 fuzzy logic controller}

Computational complexity on T2FLS is more intensive than that of T1FLS counterparts. However, calculations on T2FLS have been applied to some types of applications such as pattern recognition [36], control [37], signal processing [38], time-series forecasting [39], decision making [40], finance, communication wireless, identification system [41][42].

The use of fuzzy logic as a navigation system has been successful in some previous studies [43][44][45]. However, the use of the new T2FLS was still rare [17][46][47] and it only focused on a single mobile robot. In this paper will compare the performance of IT2FLS on the swarm robot navigation system, and is devoted to real robot implementation.

The fuzzy system focuses on how to reach targets and avoid obstacles in robot navigation. T2FLS robot navigation is the same with T1FLS, i.e., detecting obstacles using distance size of proximity sensor input. However, the difference in T2FLS is on the sensor detection. In T2FLS, after detecting an obstacle, the sensor will detect the upper and lower limits of the obstacles based on the area of the sensor used. Each obstacle will have two distinct boundaries, namely "real limits" and "safe boundaries." As long as it avoids obstacles, the barrier limit is magnified to ensure security. This expanded boundary is named a "safe limit." The robot detects true boundary barriers (real limits). Then the safe boundary of the obstacle is determined by the calculation of the controller performed in the navigation algorithm.

Paper [46] discussed mobile robot navigation using a type-2 fuzzy system. Basically, fuzzy knowledge illustrated the relationship between long-distance input and output signals that determine the angle turn of the robot.

Compared with T1FLS, the other main difference from the T2FLC, to convert the output of the fuzzy inference engine (type-2 sets) into a type-1 set needs and extra type-reducer, so the defuzzifier can process it to give a crisp output. Unluckily, the type-reducers that already exist will make computation be intensive, therefore it rendered T2FLC as an unsuitable controller for certain real-time applications.

A structure of T2FLC is described in Fig. 2. The structure is a schema of five components, namely fuzzifier, rule base, inference engine, type-reduction, and defuzzifier. In the T2FLC, T2FLSs represents inputs and/or outputs, and operate as detailed: crisp inputs, input sensors receive data, then fuzzified into input T2FLSs, which then activates an inference engine that uses the same rules are used in a T1FLC order to result from output T2FLSs. These are processed by a type reducer that projects the T2FLSs into a type 1 (this stage is called type reduction) [48][49] after which that T1FLS is defuzzified and produce the crisp output that, as an example in real robot, can be used as the command to an actuator in the control system. The defuzzification stage with the type reduction is denoted as output processing. Finally, the interval from the previous block reduced by the Defuzzifier block and acquires a single real number. 
In Fig 2, Fuzzification has a non-zero membership function that splits the crisp input into the fuzzy logic set. Therefore, the fuzzifier maps the correct entries into the fuzzy type-2 set. The inference step calculates the fuzzy output of the input and fuzzy input rules. Furthermore, the output from the fuzzy is stored in a dampening stage of type and converted to a reduced set- 1 type. Subsequent to the type of reducer, type- 1 sets are defuzzed at the defuzzifier step and the proper output for navigation.

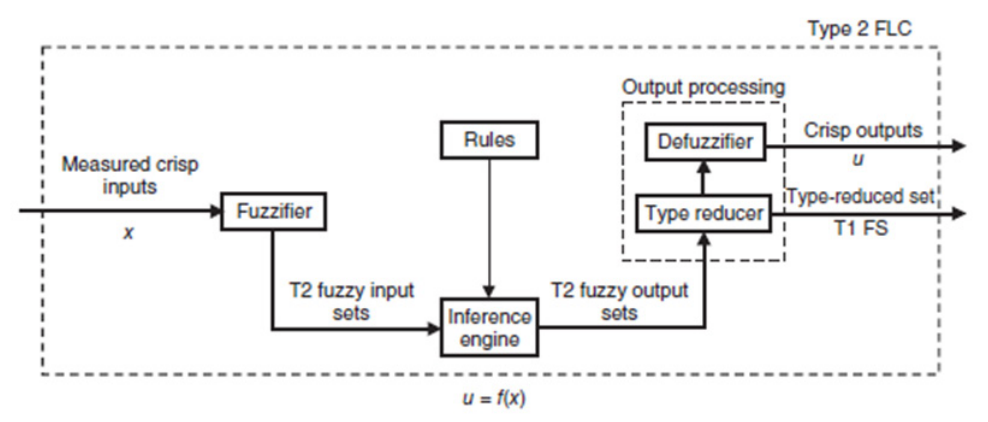

Fig. 2. Structure of Interval Type-2 Fuzzy Logic Controller

\section{Comparison between Type-1 Fuzzy Logic And Interval Type- 2 Fuzzy Logic System}

In this section, the comparison between T1FLS and IT2FLS will be shown.

\subsection{Membership function}

The important thing to design fuzzy logic controls for real speed mobile robots that use fuzzy controls in inputs and outputs is to meet the control objectives. The membership function in type-1 fuzzy sets defined for a concept.

In [7] a T2FLS, to reduce the computational cost structure is proposed. But, the computational costs are still higher even though they have the same number of input MF. In paper [12], the ITFLS2 uses to control the robot's velocity and angular velocity. A membership function from the fuzzy, are used to represent the velocity, angular velocity from the dynamic obstacles and uncertainty in position. Static and dynamic obstacles in an uncertain environment, used in the research. The results of research show that, the movement of mobile robot faster than a dynamic obstacle in this environment.

\subsection{Fuzzy inference system applications}

The mechanism in T2FLS fuzzy inference is similar to conventional on T1FLS. In combining the rule inference engine and generating a mapping of some fuzzy input sets to the T2FLS output of fuzzy set [50]. In figure 3, fuzzy inference engine blocks by mapping from the IT2FSs input to the IT2FSs output [51]. In this process, there are 
several antecedents in the rule that connect using the meet operation. The membership grade in the input set is combined in the output set using the comprehensive sup-star composition [50][52].

Mamdani [53] and Sugeno type [54] are the main two types fuzzy inference proccess. In an article [55], the author has used the Takagi-Sugeno fuzzy behaviorbased control system, which helps navigate autonomous three-wheeled mobile robots in a noisy environment.

The Mamdani FISs type, fuzzy sets are generated from the implications for each rule that is collected using maximum before applying defuzzification. On the other hand, in a typical Sugeno FIS is either weighted average or weighted sum. So, in the defuzzification process between type Mamdani and Sugeno FIS it was completely different [54].

Way the results of crips are generated from fuzzy inputs are the most basic difference between Mamdani Type FIS and Sugeno FIS. While Mamdani FIS uses a fuzzy output defuzzification technique, Sugeno FIS uses a weighted ordinary to calculate output crips [56].

The Sugeno type is more responsive because it approves more parameters in the output. Because the output is an input function, it expresses a more explicit relationship between them. Exuberance type Sugeno is in computation and more effective because of defuzzification process more strong when in the availability of noisy input data such as data sensor [54].

In work [57], has succeeded in applying a real mobile robot in a navigation system, using the fuzzy controller. It uses one fuzzy controller for navigation and barrier avoidance. Sugeno model is also used for its implementation performances. While in research conducted [33] using a combination of Takagi-Sugeno and Mamdani types, in assisting the mobile robot to achieve its objectives with evasion obstacles. TakagiSugeno to achieve its goal, as well as Mamdani type to control the speed of the robot motor.

\subsection{Rule based}

The difference between fuzzy sets type- 1 and interval type- 2 is related to the next membership function, which is when creating a rule base. Rule base from the type-1 and from the type- 2 are the same. Rule base does not depend on the based fuzzy of the set. In T2FLS has antecedents and consequent membership function. In T2FLS has antecedents and consequent membership function [50].

A type- 2 set is an output from the type- 2 inference engine. Using extension fundamental, type-1 defuzzification can produce a sharp output from the T1FLS circuit. Similarly, for T2FLS, this operation produces from type-2 sets into the type-1 sets called type-reduction.

\subsection{Type reduction}

At hierarchical of IT2FLS shows that a type-reduction is placed afterward the inference engine. The computational cost of type reduction in IT2FLS is an important 
problem [58]. Therefore, in the stage of the inference engine and the type-reducer must process interval firing strengths. It is increase computational loads and the difficulty of using IT2FLS in certain real-time applications [59].

Therefore, in the stage of reduction and deffuzzification, the need for strategies to reduce computing and resource loads. One of the strategies that have been used is the reduction in the complicatedness of hardware and software (in the case of real robot implementations) in order to pledge the fastest execution on fuzzy inference [60].

Wu dan Mendel [61] introduces a method for the type-reduced with the associated inside and outside / by the inner and outer bound sets. However, this method requires exercise data to minimize the risk function to achieve the same output with the reduced-output type. But, this data is hard to come by for mobile robots who discover a lot of sudden circumstances and will navigate around most in unfamiliar surroundings [62].

The most popular of Type-reduction is Karnik-Mendel Algorithms [50], this is computationally intensive. There are several more efficient type-reduction algorithms, and also improve, such as the Enhanced Karnik-Mendel Algorithm (EKMA) [63] and an Iterative Algorithm With Stop Condition (IASC) [64].

In a standard computing platform, considering the statistical analysis of the computational time is important, due to unknown internal delays can not be prevented.

\subsection{Defuzzification}

There are five methods of defuzzification, namely the central centroid or the center of gravity, the center line, the smallest maximum, the maximum maximum, the maximal average, which is often used in fuzzy modeling.

Ulu et al. [65] proposed a dynamic defuzzification method using a linear combination of centroid boundaries to improve the control response. But, since centroid limits are still calculated using KMA, the dynamic defuzzification method has the same computational load as the general defuzzification method.

In several studies that have been done, it can be concluded that IT2FLS can improve the deficiency of T1FLS, in spite of noise and uncertainty.

\subsection{Result}

In this research, a methodology that allows individual swarm robots to complete tasks in environments with the ability to avoid obstacles is the scope of navigation in the swarm robot. Based on the research that has done, swarm robot developed uses fuzzy logic control. The fuzzy control system as a rear wheel drive consisting of two rear wheels, namely the right and left rear wheels. Each individual robot uses a proximity sensor to measure the distance of the obstacles and the distance between the robots, as well as the surrounding destinations and identify targets. The parameters used are front obstacle distance, left obstacle distance, right obstacle distance, and find the control angle. The distance between obstacles and robots is considered safe 
for a certain distance and after that begins to deviate from the original path, this can be considered a kind of repulsion.

The default parameter values for the fuzzy type 1 input membership function use the values in Table. 1, namely as follows:

Table 1. Membership Function Input Fuzzy Type 1

\begin{tabular}{|l|c|}
\hline \multicolumn{1}{|c|}{ Membership Function } & Parameter (a, b, c, d) \\
\hline Near & $(-5,0,40,70)$ \\
\hline Far & $(40,70,100,110)$ \\
\hline
\end{tabular}

While the default value for the IT2FLS output membership function parameter uses the values in Table 2, as follows: default parameter values for the fuzzy type 1 input membership function use the values in Table. 2, namely as follows:

Table 2. Membership Function Input Fuzzy Type 1

\begin{tabular}{|c|l|c|}
\hline Output & \multicolumn{1}{|c|}{ Membership Function } & Parameter \\
\hline \multirow{3}{*}{ Right PWM } & Slow & 40 \\
\cline { 2 - 3 } & Medium & 120 \\
\cline { 2 - 3 } & Fast & 250 \\
\hline \multirow{3}{*}{ Left PWM } & Slow & 40 \\
\cline { 2 - 3 } & Medium & 120 \\
\cline { 2 - 3 } & Fast & 250 \\
\hline
\end{tabular}

Parameters that will be used as input and output values in each fuzzy logic use variables in Table 3 and Table 4.

Table 3. Membership Function Input Fuzzy Type 1

\begin{tabular}{|c|c|}
\hline Membership Function & Parameter $(\mathbf{a}, \mathbf{b}, \mathbf{c}, \mathbf{d}, \Delta)$ \\
\hline \multicolumn{2}{|c|}{ Near $(-5,-5,40,70,5)$} \\
\hline Upper & $-10,-10,15,30,5$ \\
\hline Lower & $0,0,5,20,5$ \\
\hline \multicolumn{2}{|c|}{ Far $(40,70,100,110,5)$} \\
\hline Upper & $15,30,55,55,5$ \\
\hline Lower & $25,40,45,45,5$ \\
\hline
\end{tabular}

Table 4. Membership Function Input Fuzzy Type 1

\begin{tabular}{|c|l|c|}
\hline Output & \multicolumn{1}{|c|}{ Membership Function } & Parameter (a, b, c, $\Delta$ ) \\
\hline \multirow{3}{*}{ Right PWM } & Slow & $(10,25,40,10)$ \\
\cline { 2 - 3 } & Medium & $(35,70,105,10)$ \\
\cline { 2 - 3 } & Fast & $(100,180,250,10)$ \\
\hline \multirow{2}{*}{ Left PWM } & Slow & $(10,25,40,10)$ \\
\cline { 2 - 3 } & Medium & $(100,180,105,10)$ \\
\cline { 2 - 3 } & Fast & \\
\hline
\end{tabular}


Each of the parameters above can also be replaced according to the user's needs. The membership function value of input and output for the navigation rules system used in each test can be seen in Tables 1-4. After determining the default values, then T1FLS is applied to the software as follows:

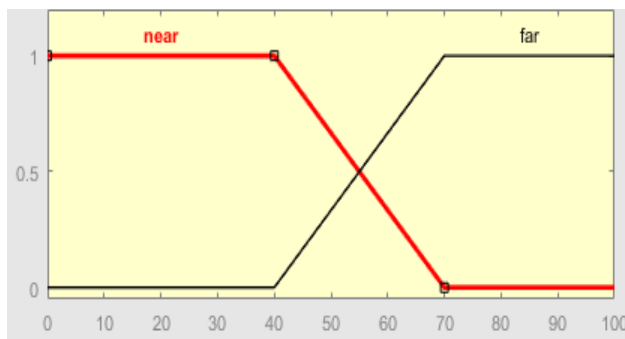

(a) Type 1 Fuzzy Logic System

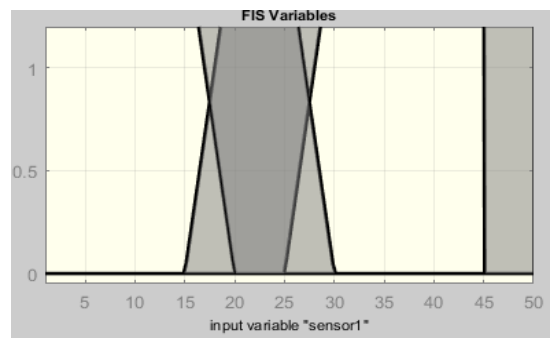

(b) Interval Type 2 Fuzzy Logic System

Fig. 3. The input of Fuzzy

Fig. 3 shows T1FLS and IT2FLS view with predefined input values on Table 6-9. On Fig. 3 a) showed one case with T1FLS with two variable linguistic as follows: Input left sensor is 50, input middle sensor is 50, and right sensor is 50 then the values of left pwm is 96.7 and right pwm is 165 . Rule base on sensor 0 is near, sensor 1 is near, and sensor 2 is near then left pwm is slow and right pwm is fast.

In Fig. 3 b), the input value is the Upper and Lower values of each membership function with the trapezoidal. And the output showed in Fig. 6. one case with IT2FLS with two variable linguistic as follows: Input left sensor is 0.5 , input middle sensor is 0.269 , and right sensor is 0.25 then the values of left pwm is 0.4663 and right pwm is 0.4663 . Rule base on sensor0 is near, sensor 1 is near, and sensor2 is near then left pwm is slow and right pwm is fast.

Fig 4, it showed that the output using gaussian with three membership function; fast, slow, and medium.

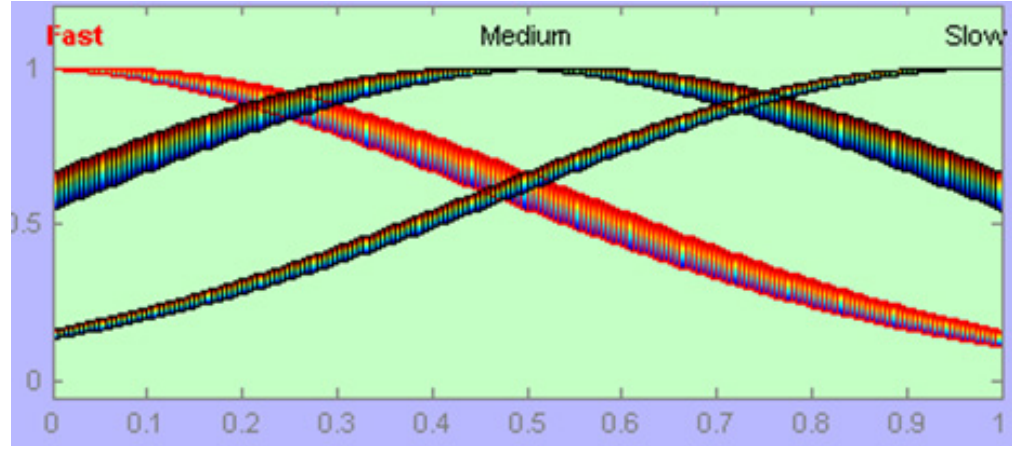

Fig. 4. The output of IT2FLS in Matlab 
The comparative performance of T1FLS and IT2FLS with control surface of the FLS shows in Fig. 5 represents the performance of FLS with the same rules.

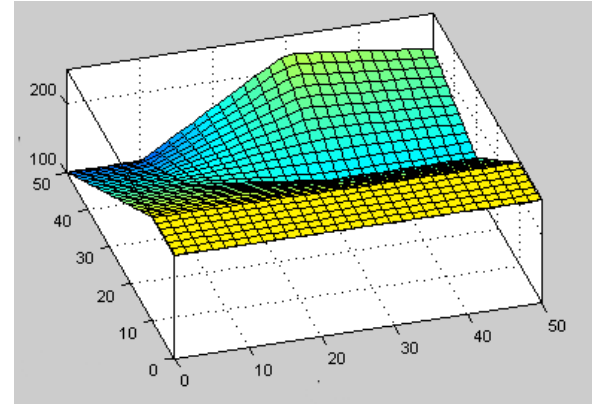

(a) TFLS1

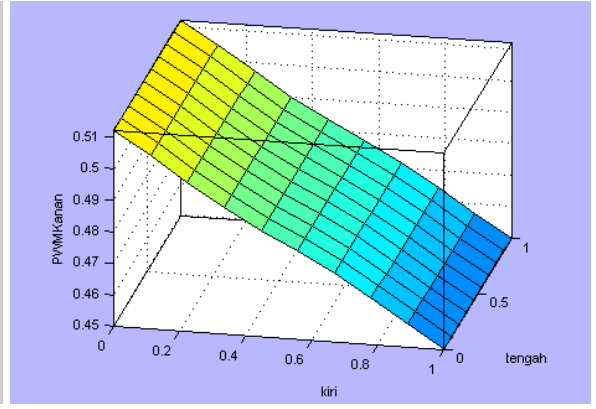

(b) IT2FLS

Fig. 5. Surface View of the FLS

There are including the control surface of fuzzy logic with 8 rules, which input is distance to the obstacle and output is speed. From In Fig. 5 a) shown that the measurement of the output changes steeply and changes in speed greatly increases. Then, in Fig. 5 b) shown that the controller with IT2FLS has a smooth surface which is characterized by the shape of the slope on the surface, where the slope represents a large decrease indicates that the velocity change drastically and smoother surface indicates better ability of T2FLS.

In each step by step in fuzzy logic computations performed using predetermined input and output values. So that from the calculation of each fuzzy logic can be applied to each individual mobile robot. In the real robot, TFLC is proposed to navigate individual mobile robots to the target. Input from TFLC is the distance between the robots to the obstacle and the robots to the robots. The output of TFLC is the left and right motor speed. TFLC implemented with a membership function for each input as illustrated in Table 1-4.

The real swarm robot experiment using TFLS1 and IT2FLS algorithms can be seen in Figure 8. The experiment was done using 3 robots with three ultrasonic sensors, one compass sensor and one X-Bee in each robot. On the actual robot with the shape of circular, it has $15 \mathrm{~cm}$ diameter and $17 \mathrm{~cm}$ height and it using three wheels. Two wheels behind the robot have a function as a controller, and the third wheel used to make the robot move freely. By connecting the two dc motor with the two driving wheels respectively, the rotation direction of each motor controlled by the direction of a drive current of the dc motor and duty cycle of Pulse Width Modulation (PWM), controls the rotation speed of the driving wheels.

This experiment compared the performance of swarm robot using T1FLS and IT2FLS. The result of swarm robot navigation with no obstacle shows in Fig. 7. The result is the path to the target relatively smooth. Each robot, gradually towards to the midpoint and towards to the target. Robot speed varies, fuzzy logic controller defines the angular velocity. 

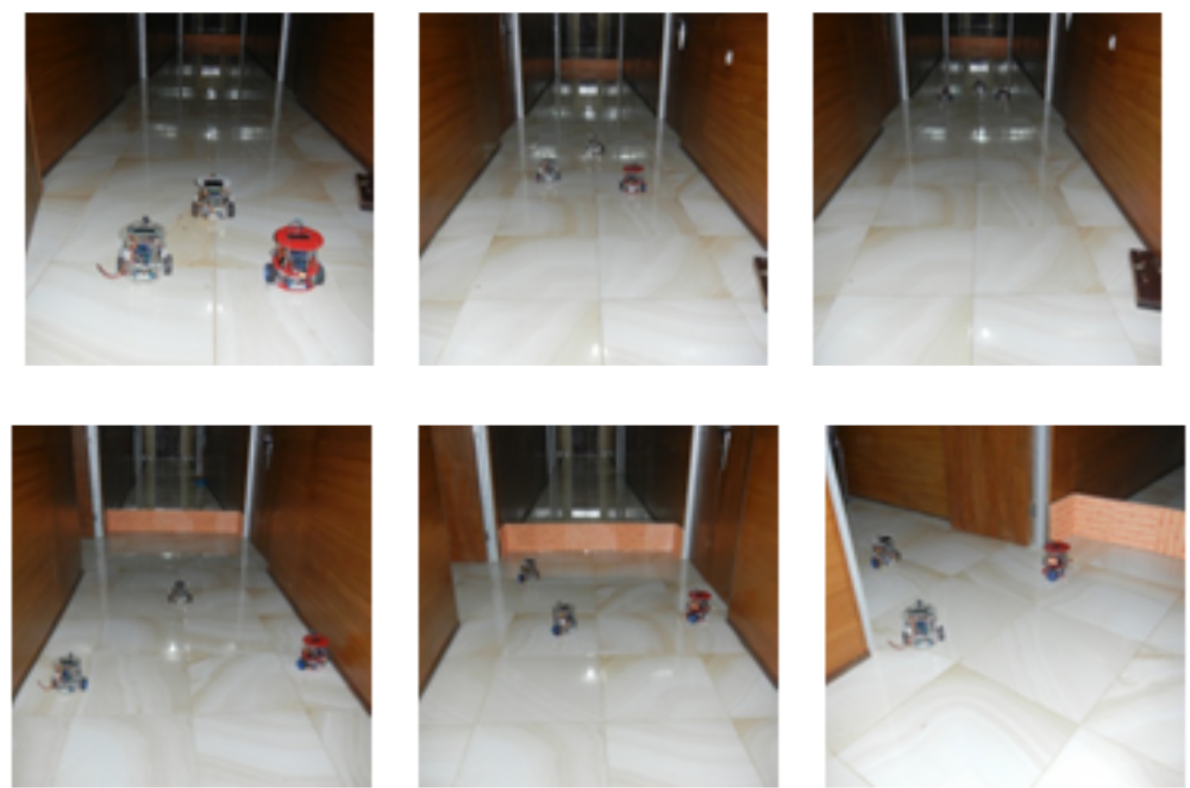

Fig. 6. Swarm Robot Navigation Experiments
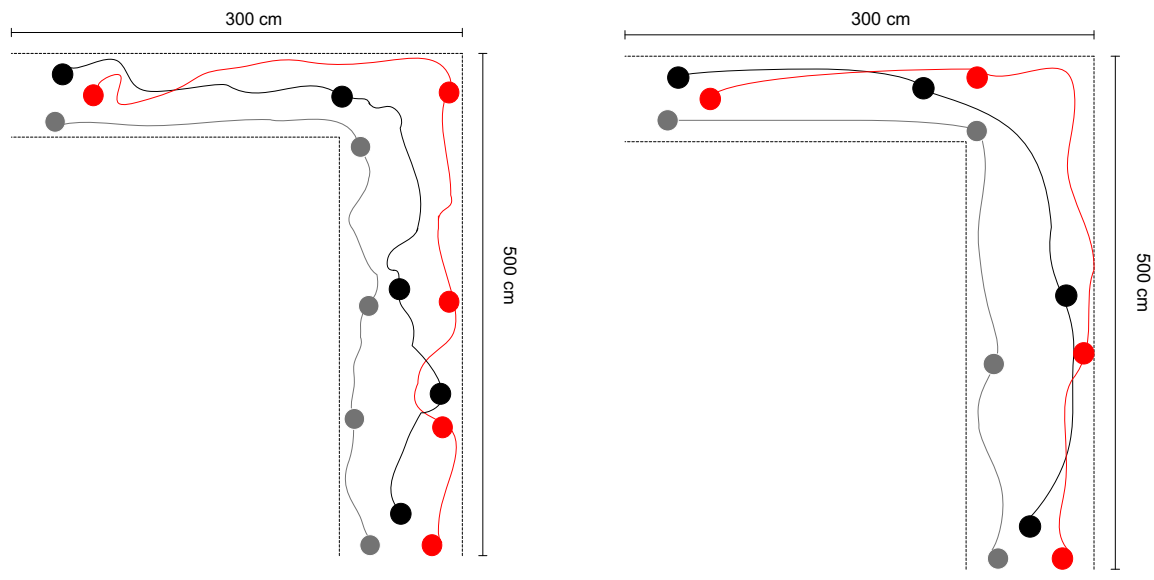

Fig. 7. Swarm Robot Navigation Using Fuzzy Logic

Experiment was done in environment without obstacle. The diferences robot movement between T1FLS and IT2FLS are IT2FLS moves more slowly due to the type-reduction process and more time spending than T1FLS. But, IT2FLS have better and stronger abilities for path planning, avoiding obstacles and in navigation, compared to T1FLS 


\section{Conclusion}

This paper aimed to compare type- 1 and type-2 fuzzy logic performance in controlling swarm robot as tools for complex problem modeling, especially for path navigation. Each has its advantages and disadvantages with some type of fuzzy logic system. In general, the Type 2 Fuzzy Logic System (T2FLS) has better performance rather than the Type-1 Fuzzy Logic System (T1FLS). T1FLS is much faster than others, particularly when considering Real Time apps and easier to design when compared to T2FLS, but has no resistance to interference, and does not support some degree of uncertainty. At T2FLS though it is more complex computation than T1FLS but effective in handling uncertainty. In resolving the problem of uncertainty, Type-2 Fuzzy Logic System (T2FLS) has been used in modeling uncertainties in solving complex problems as well as improving accuracy.

Based on the evaluation of this research, there are three input sensor that we used. Each sensor should achieve the task of moving to the destination and avoid obstacles. Thus, the robot has three tasks: avoiding obstacles, moving to the destination, as well as keeping by avoiding collisions among robots. The environment used is an environment without obstacles with different arena shapes and sizes. In an environment without obstacles, there was no disturbance effects occured. If the robot was closed that obstacles, the robot had to stay away to avoid a collision. This research used three swarm robots with its each tasks. If the robot was far from that group, then the robot would move towards one another to defend the swarm. If each robot was closed, the robot had to stay away one another to avoid a collision.

Based on the results of research that has been done by some researchers shown that the controller IT2FLS provide better results than T1FLS. Due to the nature of the T2 fuzzy sets, a T2FLS is more complicated than a T1FLS. Speaking of theory, any type can be the functions in the rule consequent; but, using the linear functions is the vast majority of the fuzzy systems. Utilization nontrapezoidal or nontriangular input fuzzy sets can minimize configuration for the typical TS fuzzy systems, smaller configuration as compared to the general Mamdani fuzzy systems as the results.

In the real robot experiment which used 3 robots, the diferences robot movement between T1FLS and IT2FLS are IT2FLS moves more slowly due to the typereduction process and more time spending than T1FLS. But, IT2FLS have better and stronger abilities for path planning, avoiding obstacles and in navigation, compared to T1FLS

\section{$5 \quad$ References}

[1] P. Benavidez et al., "Multi-domain robotic swarm communication system," 2008 IEEE Int. Conf. Syst. Syst. Eng. SoSE 2008, 2008. https://doi.org/10.1109/SYSOSE.2008.4724189

[2] B. Sun and J. Li, "Multi-Robot Cooperation Strategy Based on Wireless Sensor Network," Int. J. Online Biomed. Eng., vol. 14, no. 11, pp. 77-89, 2018.

[3] J.-H. Lee and C. W. Ahn, "Improving Energy Efficiency in Cooperative Foraging Swarm Robots Using Behavioral Model," 2011 Sixth Int. Conf. Bio-Inspired Comput. Theor. Appl., pp. 39-44, 2011. https://doi.org/10.1109/BIC-TA.2011.69 
[4] B. Tutuko, S. Nurmaini, Saparudin, and G. F. Fitriana, "Enhancement of Non-Holonomic leader-follower formation using Interval Type-2 Fuzzy Logic Controller," Int. J. Online Eng., vol. 14, no. 9, pp. 124-142, 2018. https://doi.org/10.3991/ijoe.v14i09.8568

[5] O. S. and E. S, ahin, "Probabilistic Aggregation Strategies in Swarm Robotic Systems," METU-CENG-TR-2005-02, 2005.

[6] A. Vardy and G. Vorobyev, "Cache consensus : rapid object sorting by a robotic swarm," pp. 61-87, 2014.

[7] J. Werfel, "Collective Construction with Robot Swarms," Morphog. Eng. Springer, pp. 115-140, 2012. https://doi.org/10.1007/978-3-642-33902-8 5

[8] M. Algabri, H. Mathkour, H. Ramdane, and M. Alsulaiman, "Comparative study of soft computing techniques for mobile robot navigation in an unknown environment," Comput. Human Behav., vol. 50, pp. 42-56, 2015. https://doi.org/10.1 016/i.chb.2015.03.062

[9] D. Di Paola, A. Milella, G. Cicirelli, and A. Distante, "An Autonomous Mobile Robotic System for Surveillance of Indoor Environments," vol. 7, no. 1, pp. 19-26, 2010.

[10] R. Paper, M. Faisal, R. Hedjar, M. Al Sulaiman, and K. Al-mutib, "Fuzzy Logic Navigation and Obstacle Avoidance by a Mobile Robot in an Unknown Dynamic Environment," 2013.

[11] A. Hossain and I. Ferdous, "Autonomous robot path planning in dynamic environment using a new optimization technique inspired by bacterial foraging technique," Rob. Auton. Syst., vol. 64, pp. 137-141, 2015.

[12] S. Junratanasiri, S. Auephanwiriyakul, and N. Theera-Umpon, "Navigation system of mobile robot in an uncertain environment using type-2 fuzzy modelling," 2011 IEEE Int. Conf. Fuzzy Syst. (FUZZ-IEEE 2011), pp. 1171-1178, 2011. https://doi.org/10. 1109/FUZZY.2011.6007722

[13] W. L. Xu and S. K. Tso, "Sensor-based fuzzy reactive navigation of a mobile robot through local target switching," IEEE Trans. Syst. Man Cybern. Part C Appl. Rev., vol. 29, no. 3, pp. 451-459, 1999.

[14] X. Chen and Y. Li, "Smooth Formation Navigation of Multiple Mobile Robots for Avoiding Moving Obstacles," Int. J. Control. Autom. Syst., vol. 4, no. 4, pp. 466-479, 2006.

[15] A. Over and F. Sets, "The Concept of a Linguistic Variable and its Application to Approxirqate Reasoning-III*," vol. 80, pp. 43-80, 1975.

[16] J. Bo, Y. Wang, and N. Xu, "Study of wireless sensor network route based on improved ant colony algorithm," Int. J. Online Eng., vol. 12, no. 10, pp. 86-90, 2016 https://doi.org/10.3991/ijoe.v12i10.6196

[17] S. Nurmaini, S. Zaiton, and D. Norhayati, "An embedded interval type-2 neuro-fuzzy controller for mobile robot navigation," Conf. Proc. - IEEE Int. Conf. Syst. Man Cybern., no. October, pp. 4315-4321, 2009. https://doi.org/10.1109/ICSMC.2009.5346800

[18] S. N. Hassan and S. Deepika, "Fuzzy based Mobile Robot Navigation in an Unknown Environment," in In Paper presented at the international conference on computing, 2012, p. 2012.

[19] R. Martinez-Soto, O. Castillo, L. T. Aguilar, and A. Rodriguez, "A hybrid optimization method with PSO and GA to automatically design Type-1 and Type-2 fuzzy logic controllers,” Int. J. Mach. Learn. Cybern., vol. 6, no. 2, pp. 175-196, 2015.

[20] X. Wei, "Robot path planning based on simulated annealing and artificial neural networks," Res. J. Appl. Sci. Eng. Technol., vol. 6, no. 1, pp. 149-155, 2013. https://doi.org/10.19026/rjaset.6.4050 
[21] Y. Wang, D. Tian, and Y. H. Li, "An Improved Simulated Annealing Algorithm for Travelling Salesman Problem,” Int. J. Online Biomed. Eng., vol. 9, no. 4, pp. 28-32, 2013. https://doi.org/10.3991/ijoe.v9i4.2822

[22] P. Mohanty and D. Parhi, "Controlling the Motion of an Autonomous Mobile Robot Using Various Techniques: a Review,” J. Adv. Mech. Eng., pp. 24-39, 2013.

[23] M. A. P. Garcia, O. Montiel, O. Castillo, R. Sepúlveda, and P. Melin, "Path planning for autonomous mobile robot navigation with ant colony optimization and fuzzy cost function evaluation,” Appl. Soft Comput., vol. 9, no. 3, pp. 1102-1110, 2009. https://doi.org/10.1 016/j.asoc.2009.02.014

[24] J. T. Starczewski, "Efficient triangular type-2 fuzzy logic systems," Int. J. Approx. Reason., vol. 50, no. 5, pp. 799-811, 2009. https://doi.org/10.1016/j.ijar.2009.03.001

[25] R. Sepulveda, O. Castillo, P. Melin, a. Rodriguez-Diaz, and O. Montiel, "Handling Uncertainty in Controllers Using Type-2 Fuzzy Logic," 14th IEEE Int. Conf. Fuzzy Syst. 2005. FUZZ '05. pp. 237-262, 2005.

[26] S. K. Pradhan, D. R. Parhi, and A. K. Panda, "Fuzzy logic techniques for navigation of several mobile robots," Appl. Soft Comput. J., vol. 9, no. 1, pp. 290-304, 2009. https://doi.org/10.1016/j.asoc.2008.04.008

[27] H. Hagras and C. Wagner, "Towards the Wide Spread Use of Type-2 Fuzzy Logic Systems in Real World Applications," no. July, pp. 14-24, 2012.

[28] D. Wu and W. W. Tan, "A simplified type-2 fuzzy logic controller for real-time control." ISA Trans., vol. 45, no. 4, pp. 503-516, 2006. https://doi.org/10.1016/S0019$\underline{0578(07) 60228-6}$

[29] D. A. Pomerleau, "Efficient training fo artificial neural networks for autonomous navigation," vol. 3, no. 88-97, pp. 88-97, 1991.

[30] P. Rusu, E. M. Petriu, T. E. Whalen, A. Cornell, and H. J. W. Spoelder, "Behavior-based neuro-fuzzy controller for mobile robot navigation," IEEE Trans. Instrum. Meas., vol. 52, no. 4, pp. 1335-1340, 2003. https://doi.org/10.1109/TIM.2003.816846

[31] R. H. Abiyev, B. Erin, and A. Denker, "Navigation of Mobile Robot Using Type-2 Fuzzy System," pp. 15-26, 2017. https://doi.org/10.1007/978-3-319-63315-2 2

[32] M. Faisal, R. Hedjar, M. Al Sulaiman, and K. Al-Mutib, "Fuzzy logic navigation and obstacle avoidance by a mobile robot in an unknown dynamic environment," Int. J. Adv. Robot. Syst., vol. 10, 2013.

[33] P. Gupta, S. Pandey, and A. Pandey, "Intelligent navigation and control of a mobile robot in static and dynamic environments using hybrid fuzzy architecture," Int. J. Auton. Comput., vol. 2, no. 3, p. 255, 2017. https://doi.org/10.1504/IJAC.2017.10008072

[34] R. H. Abiyev, I. Günsel, N. Akkaya, E. Aytac, A. Çağman, and S. Abizada, "Robot Soccer Control Using Behaviour Trees and Fuzzy Logic," Procedia Comput. Sci., vol. 102, no. August, pp. 477-484, 2016. https://doi.org/10.1016/j.procs.2016.09.430

[35] S. Nadarajah and K. Sundaraj, "A survey on team strategies in robot soccer : team strategies and role description," pp. 271-304, 2013.

[36] S. Nurmaini and B. Tutuko, "Pattern Recognition Approach for Formation Control for Swarm Robotics Using Fuzzy-Kohonen Networks," no. August, pp. 19-20, 2015.

[37] R. Mart??nez-Soto, O. Castillo, and L. T. Aguilar, "Type-1 and Type-2 fuzzy logic controller design using a Hybrid PSO-GA optimization method," Inf. Sci. (Ny)., vol. 285, no. 1, pp. 35-49, 2014.

[38] O. Castillo and P. Melin, "Interval type-2 fuzzy logic applications," Stud. Comput. Intell., vol. 182, no. August, 2009. 
[39] K. Y. Degtiarev and N. V. Remnev, "Linguistic Resumes in Software Engineering: The Case of Trend Summarization in Mobile Crash Reporting Systems," Procedia Comput. Sci., vol. 102, no. August, pp. 121-128, 2016. https://doi.org/10.1016/j.procs.2016.09.378

[40] A. I. Jabbarova, "Solution for the Investment Decision Making Problem through Interval Probabilities," Procedia Comput. Sci., vol. 102, no. August, pp. 465-468, 2016. https://doi.org/10.1016/j.procs.2016.09.428

[41] P. Melin and O. Castillo, "Review article A review on type-2 fuzzy logic applications in clustering clustering,tion and pattern recognition,” Appl. Soft Comput. J., vol. 21, pp. 568577, 2014. https://doi.org/10.1016/j.asoc.2014.04.017

[42] D. Wu, "On the Fundamental Differences Between Interval Type-2 and Type-1 Fuzzy Logic Controllers," IEEE Trans. FUZZY Syst., vol. 20, no. 5, pp. 832-848, 2012. https://doi.org/10.1109/TFUZZ.2012.2186818

[43] A. S. Al Yahmedi and M. A. Fatmi, "Fuzzy Logic Based Navigation of Mobile Robots," Recent Adv. Mob. Robot. pp. 287-310, 2011. https://doi.org/10.5772/25621

[44] K. S. Sethi and S. Jacob, "NNavigation of Mobile Robot using Fuzzy Logic,"” NATIONAL INSTITUTE OF TECHNOLOGY ROURKELA, 2013.

[45] A. A. Yelane, S. B. Pawar, P. S. R. Vaidya, E. C. Engg, and S. D. C. E. Selukate, "Design Approach of a Shortest Path for Robot Navigation Using Fuzzy," pp. 3-6, 2016.

[46] Krishna Kant Pandey, Anish Pandey, A. Chhotray, and D. R. Parhi, "Navigation of Mobile Robot Using Type-2 FLC," in Proceedings of the International Conference on Signal, Networks, Computing, and Systems, 2016, pp. 137-145. https://doi.org/10.1007/978-81322-3589-7 14

[47] H. a. A. Hagras, "A hierarchical type-2 fuzzy logic control architecture for autonomous mobile robots," IEEE Trans. Fuzzy Syst., vol. 12, no. 4, pp. 524-539, 2004. https://doi.org/10.1109/TFUZZ.2004.832538

[48] H. Shu, S. Member, Q. Liang, S. Member, and J. Gao, "Interval Type-2 Fuzzy Logic Systems," vol. 16, no. 2, pp. 416-427, 2008.

[49] Q. Liang and J. M. Mendel, "Interval Type-2 Fuzzy Logic Systems : Theory and Design," vol. 8 , no. 5 , pp. 535-550, 2000.

[50] N. N. Karnik and J. M. Mendel, "Centroid of a type-2 fuzzy set," Inf. Sci. (Ny)., vol. 132, no. 1-4, pp. 195-220, 2001. https://doi.org/10.1016/S0020-0255(01)00069-X

[51] S. Coupland and R. John, "Geometric Type-1 and Type-2 Fuzzy Logic Systems," vol. 15, no. 1, pp. 3-15, 2007.

[52] D. Wu and J. M. Mendel, "Enhanced Karnick-Mendel Algorithms for Interval Type-2 Fuzzy Sets and Systems,” Proc. Nafips 2007, pp. 184-189, 2007.

[53] S. Shao, "Typical Takagi-Sugeno and Mamdani Fuzzy Systems as Universal Approximators: Necessary Conditions and Comparison," pp. 824-828.

[54] A. Hamam and N. D. Georganas, "A comparison of mamdani and sugeno fuzzy inference systems for evaluating the quality of experience of hapto-audio-visual applications," HAVE 2008 - IEEE Int. Work. Haptic Audio Vis. Environ. Games Proc., no. October, pp. 87-92, 2008.

[55] L. Cherroun, N. Hadroug, and M. Boumehraz, "Hybrid Approach Based on ANFIS Models for Intelligent Fault Diagnosis in Industrial Actuator," vol. 3, no. 4, pp. 17-22, 2013.

[56] J. Jassbi, S. H. Alavi, P. J. A. Serra, and R. A. Ribeiro, "Transformation of a mamdani FIS to first order sugeno FIS,” IEEE Int. Conf. Fuzzy Syst., no. June 2014, 2007. https://doi.org/10.1109/FUZZY.2007.4295331

[57] H. Omrane, M. S. Masmoudi, and M. Masmoudi, "Fuzzy Logic Based Control for Autonomous Mobile,” Comput. Intell. Neurosci. vol. 2016, pp. 1-10, 2016. https://doi.org/10.1 $\underline{155 / 2016 / 9548482}$ 
[58] D. Wu, "Approaches for Reducing the Computational Cost of Interval Type-2 Fuzzy Logic Systems: Overview and Comparisons," IEEE Trans. Fuzzy Syst., vol. 21, no. 1, pp. 1-1, 2012. https://doi.org/10.1109/TFUZZ.2012.2197754

[59] D. W. D. Wu and W. W. T. W. W. Tan, "Computationally Efficient Type-Reduction Strategies for a Type-2 Fuzzy Logic Controller," 14th IEEE Int. Conf. Fuzzy Syst. 2005. FUZZ '05. pp. 353-358, 2005.

[60] O. Castillo, W. Pedrycz, and J. Kacprzyk, Evolutionary Design of Intelligent Systems in Modeling, Simulation and Control, vol. 257. 2009.

[61] Hongwei Wu and J. M. Mendel, "Uncertainty bounds and their use in the design of interval type-2 fuzzy logic systems," IEEE Trans. Fuzzy Syst., vol. 10, no. 5, pp. 622-639, 2002. https://doi.org/10.1109/TFUZZ.2002.803496

[62] H. A. Hagras, "A Hierarchical Type-2 Fuzzy Logic Control Architecture for Autonomous Mobile Robots," vol. 12, no. 4, pp. 524-539, 2004.

[63] D. Wu and J. M. Mendel, "Enhanced Karnik - Mendel Algorithms," IEEE Trans. Fuzzy Syst., vol. 17, no. 4, pp. 923-934, 2009. https://doi.org/10.1109/TFUZZ.2008.924329

[64] M. Melgarejo, "A fast recursive method to compute the generalized centroid of an interval type-2 fuzzy set," Annu. Conf. North Am. Fuzzy Inf. Process. Soc. - NAFIPS, pp. 190194, 2007.

[65] C. Ulu, M. Giizelkaya, and T. Eksin, "A Dynamic Defuzzification Method for Interval Type-2 Fuzzy Logic Controllers,” pp. 318-323, 2011. https://oi.org/10.1 $\underline{\text { 109/ICMECH.2011.5971303 }}$

\section{Authors}

Ade Silvia Handayani is active in teaching and having researches, especially in the area of multimedia system and artificial intelligent, Politeknik Negeri Sriwijaya. Now, she is having her doctoral degree at Sriwijaya University of Engineering department. Her current research interests are telecommunication and artificial intelligent.

Nyayu Latifah Husni is active in teaching and having researches, especially in the area of robotics of control engineering and artificial intelligent, Politeknik Negeri Sriwijaya She got various of financial supports for her research in air quality monitoring and odor localization using swarm robots area. Now, she is having her doctoral degree at Sriwijaya University of Engineering department.

Siti Nurmaini is now being active in intelligent system research at computer scence department, Universitas Sriwijaya. Her research areas are machine learning, deep learning, robotic and control, and bioinformatics.

Irsyadi Yani, joined the Department of Mechanical Engineering, Universitiy of Sriwijaya, as a Lecturer. His current research interests include Artificial Intelligence, Automatic Sorting System, and Finite Element Analysis.

Article submitted 2019-01-02. Resubmitted 2019-03-02. Final acceptance 2019-03-03. Final version published as submitted by the authors. 